\title{
Phytochemical diversity of Clinacanthus nutans extracts and their bioactivity correlations elucidated by NMR based metabolomics
}

\begin{abstract}
Clinacanthus nutans is a medicinal herb and a traditional remedy for herpes viral infection, cancer, and diabetes mellitus. Despite its popular use, there is limited information on the chemical constituents and their relationship with the bioactivities of the herb. The choice of drying and extraction methods will greatly influence the metabolite profile and the bioactivities of an herbal extract. In order to maximize retention of the original chemical profile of the herb and quality assurance, optimization of processing methods is needed. Using nuclear magnetic resonance (NMR) based metabolomics approach, we have carried out a discriminative analysis of the metabolite profiles of the leaves and stems of the herb when different drying (air, oven, and freeze) and extraction (soaking and sonication) methods were used and correlated the metabolite profiles with the total phenolic content (TPC), antioxidant and $\alpha$-glucosidase inhibitory activities. Identification of primary and secondary metabolites was performed using 1D- and 2D-NMR techniques as well as ultra-performance liquid chromatography-tandem mass spectrometry (UPLC-MS/MS). Results showed that the leaf extracts, which were richer in phenolic compounds and terpenoids, showed significantly higher bioactivities compared to the stem extracts. Several newly reported compounds in the herb, identified using tandem mass spectrometry, included gendarucin A, a gendarucin A isomer, 3,3-di-O-methylellagic acid, ascorbic acid, and two isomeric oxoprolinates. From the NMR metabolomics analysis, the PLS biplot model indicated that the presence of some phenolics compounds, terpenoids, and sulfurcontaining glucosides in the oven and air dried leaf extracts are the main components responsible for the antioxidant and $\alpha$-glucosidase inhibitory activities. This study has provided additional information on the chemistry and biology of the herb that may be useful in future development phytomedicinal preparations of C. nutans.
\end{abstract}

Keyword: Antioxidant; $\alpha$-Glucosidase inhibitory; UPLC-MS/MS; Metabolite profiling; Multivariate data analysis 